Untersuchungen und Ideen des Autors abgefaßt sind. Sie fügen sich geschickt zu diesem Zweck zusammen; nur bei den Gegenständen, die Loeb am meisten beschäftigt haben, wie künstliche Parthenogenese und Tropismen, kommt es $\mathrm{zu}$ einigen Wiederholungen - nicht unerfreulichen im Ganzen, da der Verfasser den Tatsachen ständig neue Seiten abgewinnt und neue Verknüpfungen schafft. Im allgemeinen sind es dieselben Gegenstände, wie in den "Vorlesungen über die Dynamik der Lebenserscheinungen" (Leipzig I906), die behandelt werden. Aber neue Experimente und Betrachtungsweisen sind hinzugefügt, und lebhafter und ausführlicher, wie wir es von ihm gewohnt sind, setzt er sich mit wissenschaftlichen Gegnern auseinander, so daß der Leser mit abweichenden Lehrmeinungen bekannt wird. Freilich, nüchterne unparteiische Abwägung darf niemand von einer derartigen Forschernatur erwarten, bedeutend auch durch die von seltenem Optimismus und festem Glauben an mechanistische Erklärbarkeit aller Lebenserscheinungen diktierte Kühnheit, mit der er es unternahm, die vitalsten der vitalen Vorgänge, Reizbarkeit und Entwicklungsanregung, mit den primitivsten Mitteln des Experimentators zu ergründen und sogar zu lenken, in einer Zeit, wo außer ihm wohl keiner an so einfache Zusammenhänge gedacht hätte.

Es ist nicht zu verwundern, wenn die großen erzielten Erfolge ihm selbst als Vorboten des nahen endgültigen Triumphs der mechanistischen Betrachtungsweise gegenüber allen Lebensproblemen, auch den psychischen, gelten, wie er es in dem ersten Essay unsres Buches, an den Hamburger Monistenkongreß gerichtet, in programmatischer Form ausspricht. Wer anders urteilt, kann hier immerhin die stärksten Argumente dieses Glaubens, und zugleich einen seiner erfolgreichsten Verfechter kennen lernen.

Brüel-Halle.

Plate, L. Vererbungslehre. Mit besonderer Berücksichtigung des Menschen, für Studierende, Ärzte und Züchter. Leipzig I9I3. 5 I9 S.

Als Band 2 der Handbücher der Abstammungslehre ercheint hier ein weiteres Buch, welches, sich in eingehender Weise und auf breiter Basis mit der Vererbungslehre beschäftigt. Verf. gehört zu den nicht zahlreichen Forschern, welche aus der Zeit der rein theoretischen oder auf vergleichende Anschauung gegründeten Entwicklungsforschung in das moderne Lager der experimentellen Vererbungslehre übergetreten sind und auch auf diesem Gebiete eigene, bedeutsame Untersuchungen ausgeführt haben. Man spürt das auf jeder Seite seines Buches. Die modernen exakten Ergebnisse sind in die Theorie hineingestellt und durch Hypothesen verflochten. Es ist nicht zu verkennen, daß diese Art und Weise der Darstellung die sonst hier und da etwas trockene Materie der experimentellen Daten in außerordentlicher Weise belebt. Ja ich möchte das Platesche Buch fast als das anziehendst geschriebene der modernen Vererbungsbücher bezeichnen. Damit folge ich allerdings ganz und gar nicht der Ansicht Plates selbst, welcher solchen Büchern, in denen diese theoretische Verknüpfung in den Hintergrund tritt, wie z. B. in dem Buche Baurs (vgl. sein Referat im Archiv f. Rass. u. Ges. Biol. IgII S. 500) einen Vorwurf daraus macht. Wir wollen uns im Gegenteil des außerordentlichen Wertes dieser Bücher, welche möglichst ausschließlich auf dem Boden sicher erarbeiteter experimenteller Daten aufbauen, bewußt sein, besonders auf einem Gebiete, auf dem der Theorie ja gewiß nie zu wenig geboten wurde. Jedenfalls aber gehört diese theoretische Verknüpfung zu den Hauptcharaktern des hier zu besprechenden Buches 
und damit sind Vorzüge aber auch Nachteile desselben schon zu einem großen Teile gekennzeichnet. Zugleich ist diesem Buche aber damit auch schon der besondere Platz unter den übrigen modernen Vererbungsbüchern angewiesen, in denen, vielleicht mit Ausnahme des Häckerschen Buches überall die exakt experimentelle Behandlungsweise in den Vordergrund tritt.

Als ein unbedingter Vorzug des Plateschen Buches hat besonders auch für den der Vererbungslehre noch Fernerstehenden die sehr übersichtliche, bis ins einzelne gehende Gliederung zu gelten und vor allem die eingehende Beschreibung all der verschiedenen Begriffe, Benennungen und Einteilungen, welche die moderne Erblichkeitslehre mit sich gebracht hat. Denn der Hinzukommende braucht etwas Derartiges heute auf alle Fälle. Besonders erfreulich erschien Ref. das beispielsweise in dem Abschnitt über die Unterscheidung von Dominanz und Epistase, oder die unvollkommene Dominanz usw.

Ein weiteres Charakteristikum, welches durch das ganze Buch hindurch. geht, ist die Behandlung der Gene oder Faktoren als etwas endgültig Gegebenes. Ja man hat sichtlich den Eindruck, als solle man sich die Gene durchaus als körperliche Partikelchen vorstellen, wenngleich gelegentlich darauf hingewiesen wird, daß das nicht bewiesen ist. Hier kann man nun zweierlei Ansicht sein, ob das zu empfehlen ist. Es ist sicherlich wohl gut, - und das gilt für die gesamte neuere Vererbungslehre ebenso wie für Pla tes Buch im speziellen - sich die Sachen so einfach wie möglich vorzustellen, und da kann man ja für typische Fälle gut die Faktoren wie bisher beibehalten. Aber wenn man dann all die Hypothesen braucht, welche für gewöhnlich und auch in dem Plateschen Buche als etwas ziemlich Selbst. verständliches betrachtet werden, dann wird die Theorie gefährlich. Wie häufig finden wir schon Fälle, wo dieselbe Sachlage von zwei Autoren mit ganz verschiedenen Faktoren geklärt wird und wenn dann ein dritter herankommt, noch weitere Differenzen entstehen - Beispiele dafür findet jeder, der darauf achtet, in dem Plateschen Buch eine ganze Reihe. Geht das so weiter, wird die Vererbungswissenschaft aus einer exakten experimentellen aber wieder mehr und mehr $\mathrm{zu}$ einer theoretischen. Und es liegt naturgemäß gerade bei der Plateschen Darstellungsweise die Gefahr nahe, auf diesem Wege etwas weit $\mathrm{zu}$ gehen.

Ganz besonders gefahrvoll wird das aber dann, wenn die an experimentellen Untersuchungen gewonnenen Daten auf das menschliche Vererbungsgebiet ohne Experimente übergeführt werden und Plate widmet ja gerade diesen Fällen ein besonderes über roo Seiten starkes Kapitel. Man denke sich, wir rechnen heute schon bei unseren Experimenten mit Dominanz (unvollkommener und vollkommener), Epistase, polygenen Merkmalseinheiten (gleichsinnigen und ungleichsinnigen), Verkoppelung, Konditionalfaktoren, Erregungsfaktoren, Transmutatoren, Hemmungsfaktoren usw. usw. bei Experimenten, bei welchen wir das Elternmaterial rein vor uns haben und beliebige Kombinationen ausführen können; müssen wir uns da nicht fragen, ob es geboten ist, ein solches kompliziertes System schon heute auf mensch. liche Fälle hinüberzunehmen? Es ist doch ganz ohne Zweifel, daß sich hier das Tatsachenmaterial in vielen Fällen auf ganz verschiedene Weise deuten läßt. Und dann sehe ich auch hier den Vorteil solcher Deutung nicht mehr ein. Ob man sagt, das charakteristische Gen für Diabetes mellitus ist wohl als ein Hcmmungsfaktor anzusehen, welcher die Verbrennung des Zuckers, welche im normalen Zustand erfolgt, verhindert, oder ob ich einfach sage, die Verbrennung des Zuckers wird bei der Krankheit verhindert. Wir haben uns ja hier die Gene noch gar nicht herausarbeiten können und es 
ist ebenso wahrscheinlich, daß es sich hier um ein polygenes Merkmal, um ein verkoppeltes oder um überhaupt etwas anderes handelt. Wir wissen darüber eben noch nichts und Theoretisieren führt hier einstweilen auch nicht weiter. Dann aber möchte ich ein weiteres Beispiel anführen, welches Plate zitiert, um noch eine andere Einschränkung zu begründen. Wenn in einer Familie oder einer Anzahl Familien eine Krankheit in der Hälfte der Fälle auftritt, in der anderen Hälfte nicht, so ist man bei einigermaßen größerem Material schnell mit der Erklärung da: Hier liegt eine Kreuzung von RR . DR vor, infolgedessen ergibt sich das Verhältnis 50:50 und damit ist die Mendelsche Spaltung erwiesen. Das Platesche Beispiel, von welchem hier die Rede sein soll (S. 355), Diabetes insipidus, gibt aber nun auch Kranke zu Gesunden im Verhältnis von ca. 50:50, dennoch aber haben hier die Gesunden stets gesunde, die Kranken stets kranke Nachkommenschaft. Wie soll man sich aber dies mit der Mendelschen Regel erklären? Welche Hilfshypothese wird da herangezogen?

Bei soviel Schwierigkeiten möchte man wohl bei der Lektüre gerade der auf die menschlichen Fälle sich beziehenden Kapitel des Plateschen Buches nicht selten sagen: Festina lente. Es wird eine zu schnelle und allgemeine Übertragung der noch so ungeklärten komplizierten Verhältnisse auf die menschliche Vererbung gar zu leicht die Mendelei bei der derselben noch ferner stehenden Ärzteschaft im Ansehen schmälern.

Hier und da hat Plate wohl auch bei der menschlichen Vererbung dem Wunsche, die Daten der menschlichen kranken oder gesunden Konstitution auf Vererbungsverhältnisse zurückzuführen, zuviel nachgegeben. Auf S. 24 warnt Verf. zwar selbst davor, heterogene Fälle zusammenzuwerfen und dann falsche Schlüsse zu ziehen und das ist sicher nirgends mehr am Platze, als auf diesem wichtigen, aber so unsicheren Gebiet. Dennoch aber wird hier sowohl wie auch später Langlebigkeit des Menschen auf Grund statistischer Vergleiche zwischen Vater und Sohn bzw. Mutter und Tochter usw. (im Gefolge von Pearson und Ploetz) als erblich bezeichnet. Was steckt aber einmal unter Langlebigkeit für vielerlei Heterogenes, und dann liegt nicht die geringste Veranlassung vor, etwa für Langlebigkeit ein besonderes Gen anzunehmen; wir können ja hier ohne Zweifel Modifikationswerte als ausschlaggebend heranziehen. Wir können an die Wirkungen des elterlichen Soma auf die Keimzellen im Sinne günstiger Modifikation denken. Wir können aber auch annehmen, daß die Gesamtheit aller inneren Bedingungen oder auch einige derselben im Organismus zusammenwirken, die Langlebigkeit hervorzubringen, die wir doch deshalb nicht auf ein einzelnes Gen zurückzuführen brauchen. Aber gerade hier heißt es doch aufs strengste scheiden. Zudem spricht Ploetz in seiner diesbezüglichen Arbeit auch nur von vorläufigen Resultaten, nicht endgültigen Ergebnissen, wie es nach Plate scheinen könnte.

Im Anschlusse daran möchte Ref. sodann noch in Frage stellen, ob die Erbfaktoren so ohne weiteres unter die Klebsschen inneren Bedingungen fallen. Ref. hat bisher die Erbfaktoren immer mehr mit den seiner Ansicht nach keineswegs überflüssigen Potenzen in Parallele gestellt. "Der Klebssche Begriff der Potenz scheint mir hingegen überflüssig zu sein, denn daß eine Pflanze mit roten Blüten die Fähigkeit hat, einen roten Farbstoff zu bilden, ist selbstverständlich", sagt Plate. Das wohl. Aber es kann auch eine weiße Blüte die Potenz oder den Faktor haben, roten Farbstoff zu bilden, nur aber durch innere Bedingungen, welche in Beziehung zu äußeren stehen, an der Ausbildung gehindert werden, dann wäre die Potenz schon nicht mehr so selbstverständlich. 
So wäre es möglich noch mancherlei anzuführen, worin die Auffassungen von Verf. und Ref. auseinandergehen. Es soll davon aber hier abgestanden werden. Zur allgemeinen Orientierung sei nur nỏch auf einige Einzelheiten hingewiesen.

Einmal müssen wir bei der Lektüre des Buches daran denken, da $\beta$ Verf. nicht völlig der Presence- und Absence-Hypothese huldigt, sondern statt dessen, wie früher seine Grundfaktor-Supplementtheorie heranzieht, was allerdings im allgemeinen das Verständnis nicht beeinflußt. Weiter sei besonders der eigenen Theorien der gynephoren Vererbung bei Blutern, Farbenblinden usw, gedacht. SchlieBlich sei an die Auffassungen des V'erf.s in der Frage von Selektion und Vererbung erworbener Eigenschaften erinnert, von denen Ref. die erstere in gewisser Weise fortentwickelt gegen früher erscheint.

Etwas mehr Äußerliches möchte Ref. aber zum Schluß den Verf. noch bitten, nämlich für die nächste Auflage seine Literaturliste etwas mehr durchzuarbeiten. Aus verschiedenem das eine Beispiel. Sobald überhaupt in einem Vererbungsbuche, wie das ja derzeit üblich, für Ref. aber aus hier nicht auseinanderzusetzenden Gründen keineswegs selbstverständlich ist, über Pfropfbastarde gehandelt wird, so stehen da doch wohl die Arbeiten Winklers obenan. Von Winkler ist aber im Literaturverzeichnis überhaupt nichts $z$ u finden!

E. Lehmann.

East, E. M. and Hayes, H. K. Heterozygosis in Evolution and in Plant Breeding. U. S. Dept of Agric. Bur. of Plant Ind. Bull. 243. Washington Igr2. 58 Seiten und 8 Tafeln.

Die Verfasser machten umfassende Isolierungs- und Kreuzungsversuche mit Zea Mays (die bekanntlich eine typisch allogame Pflanze ist) und konstatierten dabei, daß (künstliche) Selbstbefruchtung stets von einer Abnahme der vegetativen Kraft begleitet wurde; während umgekehrt Kreuzbefruchtung zwischen Pflanzen, die von geselbsteten Eltern herstammten, eine Steigerung der Wachstumsenergie mitführte. Da die Nachkommen der geselbsteten Pflanzen zugleich mehr oder weniger große morphologische Unterschiede darboten, und da die Abnahme der vegetativen Energie nach wiederholter Selbstbefruchtung immer kleiner wurde, glauben die Verfasser, daB die Abschwächung von der durch die Selbstbefruchtung bedingten Einschränkung der Heterozygotie verursacht wurde. Sie fanden diese Ansicht durch Versuche mit typisch autogamen Arten und Varietäten von Nicotiane bestätigt, indem Kreuzung dort in vielen Fällen beträchtlich kräftigere Pflanzen als die Eltern ergab. Die weiteren Konsequenzen der Resultate werden kurz besprochen. Kajanus.

\section{Rosen, F. Die Entstehung elementarer Arten aus Hybridisation ohne} Mendelsche Spaltung. Beiträge zur Pflanzenzucht 3 IgI3. S. 89 .

Schon früher hat der Verfasser über seine Kreuzungsversuche mit Erophila verna berichtet. Es hat sich damals herausgestellt, daß während $F_{1}$ sich intermediär und einförmig zeigte, war $F_{2}$ in hohem Grade heterogen und gab unter Ioo Pflanzen kaum eine die einer anderen glich. Die Untersuchungen brachten ferner das höchst auffallende Resultat, da $B$ jede $F_{2^{-}}$ Pflanze in $F_{3}$ eine konstante einförmige Nachkommenschaft gab.

Verfasser hat daraus geschlossen, daß die Erophila-Bastarde dem Mendelschen Spaltungsgeset $z$ nicht folgen und nimmt ferner an, daß das 